\title{
Direct measurement of the transverse wakefields of tapered collimators
}

\author{
P. Tenenbaum, ${ }^{*}$ K. L.F. Bane, L. Eriksson, J. Irwin, R. K. Jobe, D. McCormick, C. K. Ng, T. O. Raubenheimer, \\ M. C. Ross, ${ }^{\dagger}$ G. Stupakov, and D. Walz \\ Stanford Linear Accelerator Center, 2575 Sand Hill Road, Menlo Park, California 94025, USA
}

\author{
D. Onoprienko \\ Brunel University, Uxbridge, Middlesex UB8 3PH, United Kingdom \\ I. Zagorodnov \\ Deutsches Elektronen-Synchrotron, Notkestrasse 85, 22607 Hamburg, Germany
}

(Received 9 January 2007; published 12 March 2007)

\begin{abstract}
We report on a recent set of measurements of the transverse wakefields from longitudinally tapered collimators. The measurements were performed with a low-emittance $1.19 \mathrm{GeV}$ beam in the SLAC linac by inserting a collimator aperture into the beam path and reconstructing the vertical deflection of the beam as a function of the vertical position of the aperture. Each collimator in the experiment was designed to present a relatively large transverse impedance and to minimize the impedance from other contributions such as resistivity. In addition, the collimator parameters were chosen to provide some insight into the scaling of the transverse geometric wakefield as a function of the collimator's geometry. A description of the experimental apparatus and the aperture design, the method of data collection and analysis, and a comparison to theoretical and numerical predictions are presented.
\end{abstract}

DOI: 10.1103/PhysRevSTAB.10.034401

PACS numbers: 29.27.Bd

\section{INTRODUCTION}

A future linear collider with a center-of-mass energy in the $0.5-5.0 \mathrm{TeV}$ range will require a beam power of at least several megawatts in order to achieve the desired luminosity of approximately $10^{34} \mathrm{~cm}^{-2} \mathrm{~s}^{-1}$. In order to make efficient use of the available luminosity, the detector which surrounds the interaction point of such a collider must be kept tolerably free of accelerator-related backgrounds such as particles with large transverse amplitudes, synchrotron radiation from large-amplitude particles in the final quadrupole magnets, muons, etc. Although precise estimates for the impact of accelerator backgrounds on the detector are not yet available, simulations have indicated that a nonGaussian "halo" containing as little as $10^{-8}$ of the total beam power can degrade the performance of a linear collider detector [1]. Such "clean" beam conditions can only be ensured through the application of mechanical devices - collimators - which scatter or absorb particles in the halo while transmitting particles in the core to the collision point. The collimators in a linear collider will also serve an additional purpose: to protect the detector from being directly struck by a beam pulse which emerges from the linac with a large transverse amplitude or energy error. Thus, even if it were possible to guarantee that the linear collider would generate no halo, it would be essential to

\footnotetext{
*Electronic address: quarkpt@slac.stanford.edu

†Permanent affiliation: Fermi National Accelerator Laboratory, P.O. Box 500, Batavia IL 60510.

${ }^{\ddagger}$ Permanent affiliation: Physics Department, Kansas State University, 116 Cardwell Hall, Manhattan, KS 65506.
}

include a set of collimators between the end of the linac and the collision point.

The post-linac collimator system in a linear collider presents a wide variety of challenges to the designers. The system must stop the vast majority of particles which would generate backgrounds in the detector; it must incorporate collimator gaps which are of physically realizable dimensions; it must preclude the destruction of the collimators themselves in the event of a direct hit by the core of the beam; and it must accomplish all of this in an acceptable length of beam line. Although collimator designs for linear colliders are now quite sophisticated, all of them essentially follow the prescription set forth by the designers of the Next Linear Collider (NLC) in 1996 [2]: a system of thick "absorber" collimators which are shadowed by thin "spoiler" collimators in a region with elevated betatron and dispersion functions, which are placed some distance from the IP. The elevated betatron and dispersion functions enlarge the beam size at the spoiler locations; this enlargement serves the dual purpose of permitting large spoiler gaps (on the order of a fraction of a millimeter) and ensuring that the beam is too large to severely damage the spoiler in the event of impact. The spoilers in turn further enlarge the beam through multiple Coulomb scattering such that the maximum power density incident on an absorber is too small to damage the absorber. The collimation system is placed some distance from the collision point in order to allow space for devices that reduce the flux of muons from the absorbers.

In addition to the constraints above, the small apertures of the collimators in a linear collider beam delivery system 
make them a potentially serious source of transverse wakefields. The collimator wakefields can cause a luminosity reduction through jitter amplification: incoming bunches which are not centered in a collimator receive a net centroid deflection. In addition, the variation of the kick along the length of a single bunch can cause a net increase in the transverse projected emittance.

\section{A. Existing measurements of collimator wakefields}

The effect of collimators with small apertures on the transverse beam dynamics was observed during the operation of the Stanford Linear Collider (SLC). Several attempts were made to estimate and to measure the severity of collimator transverse wakefields [3-5]. These measurements were not entirely satisfactory for a number of reasons: poor and variable beam quality at the collimator locations (end of the linac and/or the final focus of the SLC), inadequacy of beam measurement devices, uncertain condition of the collimators (i.e., some of the measurements were performed using collimators which had been damaged by incident beam power, although this was not generally known at the time of measurement). The most fundamental limitation of these measurements, however, was that only the collimators which were in use for SLC background-control purposes were available for measurement. There was no opportunity to perform a systematic study in which collimator surfaces, geometries, etc. could be varied and the resulting effects examined in detail.

\section{B. Review of the theory of collimator wakefields}

The transverse wakefields that arise due to a change in dimensions of the vacuum chamber at the collimator are collectively referred to as the geometric wakefields. Another component of the wake, due to the resistance of the beam pipe walls, is called the resistive-wall (RW) wakefield. For the International Linear Collider (ILC), the RW wake is an important contribution; for the experiments described here, however, it is orders of magnitude weaker than the geometric wake and will, therefore, not be considered in this report.

In this section we collect analytical formulas for the transverse wake kick of collimators that will be used to compare with the measurements. The collimators of interest are of the type sketched in Fig. 1: a symmetric collimator (symmetric both longitudinally and transversely) tapers from vertical half-gap $b_{2}$ to vertical half-gap $b_{1}$ $\left(b_{1} \ll b_{2}\right)$ and back again. The taper length is distance $L_{T}$, resulting in a taper angle $\theta_{T}=\tan ^{-1}\left[\left(b_{2}-b_{1}\right) / L_{T}\right] \approx$ $b_{2} / L_{T}$. In principle, the collimator can also have a flat region of length $L_{F}$ at the minimum half-gap size, although for all collimators used in the experiment $L_{F}=0$. Two types of collimators are used in the experiment: square (in which the horizontal and vertical half-gaps are approximately equal), and flat (in which the vertical half-gap at its
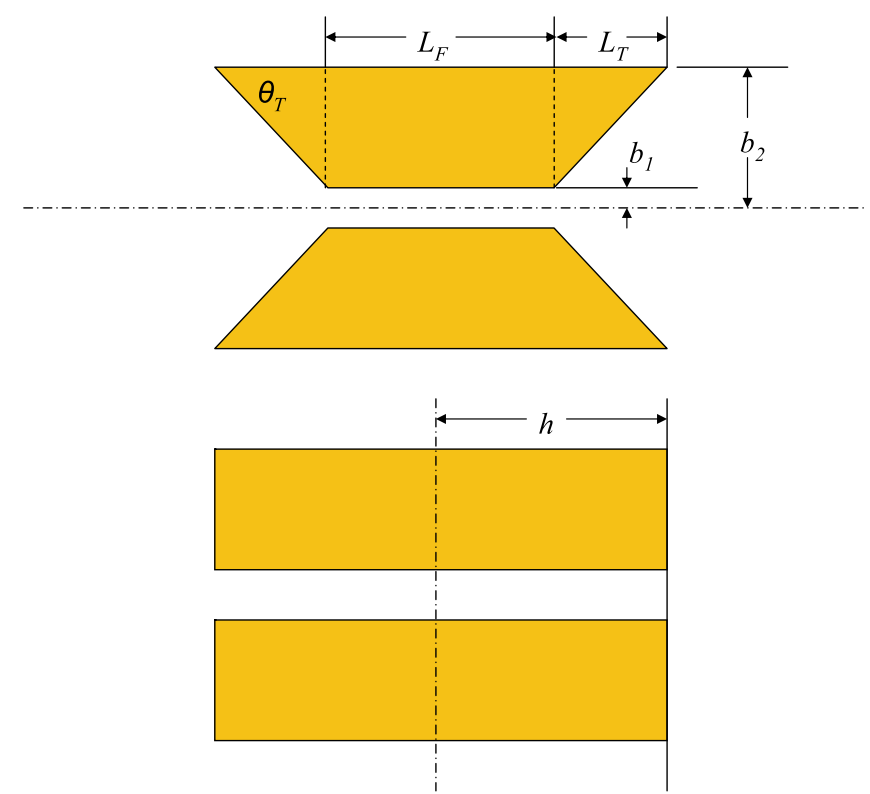

FIG. 1. (Color) Side view (top) and "beam's eye" view (bottom) of a tapered collimator. In this case, a flat collimator (rather than a round collimator) is shown.

minimum is much smaller than the horizontal half gap). In the case of a flat collimator, the half-width of the gap in the noncollimating (horizontal) direction is denoted by $h$.

For a high energy beam passing through a (symmetric) collimator at a vertical distance $y_{0} \ll b_{1}$ from the axis, the mean centroid kick is given by

$$
y^{\prime}=\frac{y_{0} Q \kappa}{E},
$$

with $Q$ the bunch charge, $\kappa$ the (vertical) kick factor-the transverse kick averaged over the length of the beam (typically reported in $\mathrm{V} / \mathrm{pC} / \mathrm{mm}$ ), and $E$ the beam energy. Thus, for small offsets from the center of the collimator, wakefields result in a linear amplification of the total bunch-by-bunch jitter of the beam.

Analytical formulas for the impedance and, equivalently, $\kappa$ can be found in the limits where the parameter

$$
\alpha \equiv \theta_{T} b_{1} / \sigma_{z}
$$

( $\sigma_{z}$ is the rms bunch length) is either small or large compared to 1 , regimes which we denote with the labels inductive and diffractive, respectively. Note that, in the ILC beam delivery system, with representative values $\sigma_{z}=$ $300 \mu \mathrm{m}, b_{1}=1 \mathrm{~mm}$, and $\theta_{T}=10 \mathrm{mr}, \alpha=0.03$, we are typically in the inductive regime. For the measurements of this report, however, we will see that typically $\alpha \sim 1$, and it is not always clear which, if any, of the analytical models apply. In these cases the analytical theory gives a result that can only serve as an order-of-magnitude estimate, and may differ from the correct value by a numerical factor. For detailed comparison with measurements we will also in- 
clude numerical calculations. In all of the following, the meter-kilogram-second-ampere system of units is used.

\section{The inductive regime}

In the inductive regime, $\alpha \ll 1$ and $\theta_{T} \ll 1$, the (transverse) wake induced kick of a bunch offset from the collimator axis is proportional to the bunch shape, and the kick factor is proportional to the inverse of the bunch length. In the case of a (symmetric) round, tapered collimator, assuming a Gaussian bunch distribution, it is given by $[6,7]$

$$
\kappa=\frac{Z_{0} c \alpha}{2 \pi^{3 / 2} b_{1}^{2}}\left(1-\frac{b_{1}}{b_{2}}\right),
$$

with $Z_{0}=377 \Omega$.

For flat collimators, when $\alpha \ll b_{1}^{2} / h^{2}$, the kick is given by $[8,9]$

$$
\kappa \approx \frac{Z_{0} c}{4 \sqrt{\pi}} \frac{\alpha h}{b_{1}^{3}},
$$

with $h$ the half-width (in the horizontal direction) of the collimator. In order-of-magnitude terms, then, the kick from a shallow tapered flat collimator is larger than the kick from an equivalent round one by a factor of $\pi h / 2 b_{1}$. Although this kick factor has the unfortunate property of diverging as $h \rightarrow \infty$, it should be noted that the applicability criterion is quite strict and prevents an unacceptable divergence of the transverse wake. A recent work by Krinsky [10] addresses the issue of the impedance in the limit $h \rightarrow \infty$ for zero frequency.

In our experiments the condition $\alpha \ll b_{1}^{2} / h^{2}$ is never met by far: the largest ratio $b_{1}^{2} / h^{2}=0.04$, is much smaller than the smallest $\alpha(=0.33)$. However, an intermediate result may apply. For the case $b_{1}^{2} / h^{2} \ll \alpha \lesssim 1$, the kick factor can be approximated by [9]

$$
\kappa \approx(1.35) \frac{Z_{0} c}{4 \pi b_{1}^{2}} \alpha^{1 / 2}
$$

Note that in [9] the kick factor calculation is wrong by a factor of 2; Eq. (5) has the correct magnitude. Furthermore, Eq. (5) considers only the dipole impedance of the flat collimator and neglects the quadrupole impedance. To our knowledge, the quadrupole contribution to the impedance of a collimator is not known in the regime of Eq. (5).

For the flat collimators, for the inductive comparison we will use Eq. (5). For the square collimator, since we have no analytical model, we will resort to using the round result, Eq. (3).

\section{The diffractive regime}

In the diffractive regime, $\alpha \gg 1$, analytical formulas exist in the limits of short and long collimators, which we distinguish by the length of the flat region at minimum aperture $\left(L_{F} \rightarrow 0\right.$ and $L_{F} \rightarrow \infty$, respectively). In all cases the (transverse) wake induced kick is proportional to the integral of the bunch shape, and the kick factor is independent of bunch length. In the case of a round, long collimator, the (dipole) kick factor is [11]

$$
\kappa=\frac{Z_{0} c}{2 \pi}\left(\frac{1}{b_{1}^{2}}-\frac{1}{b_{2}^{2}}\right) \text {. }
$$

If the collimator is short, however, the result is about half as much [12]:

$$
\kappa=\frac{Z_{0} c}{4 \pi}\left(\frac{1}{b_{1}^{2}}-\frac{b_{1}^{2}}{b_{2}^{4}}\right) .
$$

In the case of short, flat collimators, the leading order behavior (for $b_{1} \ll b_{2}$ ) is the same as given in Eq. (7) [12]. That is, for a flat collimator with $L_{F} \rightarrow 0$ we have

$$
\kappa \approx \frac{Z_{0} c}{4 \pi b_{1}^{2}} \text {. }
$$

In the special case of a square, short collimator [13],

$$
\kappa \approx \frac{Z_{0} c}{4 \pi b_{1}^{2}}\left(\frac{1}{2}+\frac{1}{\pi}\right)
$$

The last two equations will be used in comparison with the measurements in this report. The wake kick in the diffractive regime is expected to constitute an upper bound on the kick for a collimator with a given minimum half-gap $b_{1}$.

A collimator which is in the long, flat, diffractive regime is expected to produce a wake kick which is approximately the same as the kick from a long, round diffractive collimator, Eq. (6), up to a constant of order unity [13]. There are no collimators in this regime considered in this set of experiments, but we include this estimate for completeness.

\section{COLLIMATOR WAKEFIELD TEST STAND}

Figure 2 shows a schematic representation of the SLAC collimator wakefield test stand. The apparatus consists of a rectangular stainless steel vacuum chamber approximately 5 feet in length, 2 feet in width, and 1 foot in height. The top panel of the vacuum chamber can be completely removed to access the interior, and in normal operation an indium wire between the top panel and a grooved lip on the main body provides the vacuum seal. The vacuum chamber is supported by a precision vertical translation stage constructed for the final focus test beam (FFTB) [14]. This stage is capable of moving the vacuum chamber through $\pm 1.4 \mathrm{~mm}$ from its neutral position in $1 \mu \mathrm{m}$ steps.

The vacuum chamber contains an aluminum insertion approximately 5 feet long, 11 inches wide, and 3 inches tall. The aluminum insertion is fabricated in two pieces, top and bottom, which are bolted together and supported on a pair of precision-machined and dry-lubricated rails by a set of four linear bearings. The insertion is attached to an external stepper motor by a feedthrough on the side of the 


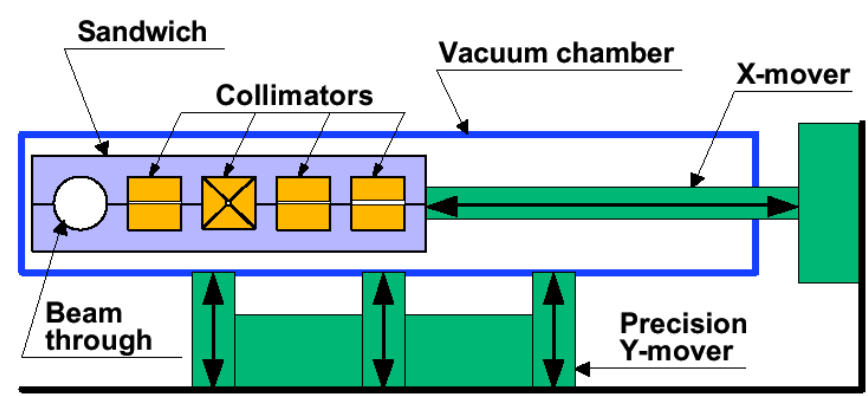

FIG. 2. (Color) Schematic end-on view of collimator wakefield test stand. A large vacuum vessel contains an aluminum insertion which is in turn filled with test apertures. During experiments, the horizontal translation stage engages an aperture in the beam path, and the precision vertical mover moves the collimator relative to the beam. Reconstruction of the resulting beam kick allows determination of the collimator kick factor. As actually constructed, the large circular aperture is on the far right rather than the far left side, so that in the event of a power failure to the horizontal translation stage the large aperture will be pushed into place by the pressure differential, rather than one of the small apertures.

vacuum chamber. The aluminum insertion contains 5 machined channels which run its full length: one channel ("slot zero") is 1.5 inches in diameter, circular, and completely regular. The other four channels are square in cross section, with width and height of 1.5 inches. A test aperture can be installed in each of the four square channels.

During normal operation, the circular aperture is positioned along the beam trajectory. When a collimator wakefield measurement is to be performed, the stepper motor moves the insertion on its support rails (in the horizontal direction) until the desired square channel is positioned in the beam path. Once this is accomplished, the vertical translation stage is used to move the entire vacuum assembly (including the collimator) in the path of the electron beam. The resulting deflection of the electron beam is measured, and thus the kick factor $\kappa$ of the collimator can be determined.

\section{A. Experimental requirements}

The SLAC accelerator complex includes a large number of beam lines and locations which could house the collimator wakefield test stand. The location which was ultimately chosen was a point immediately downstream of the damping ring injection point (DRIP), where the lowemittance beam emerges from the damping ring into the $50 \mathrm{GeV}$ linac. The location has several experimental advantages: (i) Duty cycle: all beams to PEP, the positron production target, the FFTB, or the linac tuneup dumps pass through this point, thus beam is almost always available. (ii) Beam quality: the beam quality at this location, downstream of the damping rings and upstream of the linac (with its intense wakefields), is perhaps the highest in the entire complex. (iii) Low beam energy, resulting in larger transverse deflections. (iv) Short bunches: the bunch compressor between the damping ring and the test stand permits rms bunch lengths between $0.5 \mathrm{~mm}$ and $1.5 \mathrm{~mm}$ to be used in experiments. (v) Instrumentation: 5 beam position monitors (BPMs) upstream of the test stand and 32 BPMs downstream permit the wakefield kick angle to be measured with high precision.

The principal drawback to the DRIP location is its relative inaccessibility: since all users rely on beams that pass through the DRIP, opportunities for access are rare. In addition, the criticality of the location to all programs places a premium on easing beam operations in the vicinity of the wakefield test stand. In order to address these requirements, the aluminum insertions accept up to 4 collimator apertures at one time, and one chamber is always maintained with no collimators for ease of operation when measurements are not being performed.

\section{B. Determination of the bunch length}

As shown in Sec. IB, the rms bunch length is a parameter which strongly influences the expected wakefield kick factors, and thus knowledge of the bunch length is essential for any comparison between experimental measurements and theoretical predictions. The bunch length measurement system in the SLAC linac consists of a profile monitor and a dipole-mode radio-frequency (RF) cavity in which the phase of the RF is adjusted such that the beam arrives on the zero crossing of the cavity [15]. The fields in the cavity vertically deflect the particles in the beam in proportion to their distance from the zero crossing; by measuring the rms vertical size of the beam some distance downstream of the cavity, one can calculate the rms deflection due to the cavity's fields, and thus the rms bunch length.

The rms bunch length and shape were not routinely measured during collimator wakefield studies. Instead, the bunch length was measured as a function of the RF voltage in the bunch compressor during a single set of wakefield studies, and the resulting relationship was assumed to hold during all subsequent studies. The expected accuracy of the measurement is at the level of $10 \%$, with the scale factor of the BPM used in the calibration procedure of the bunch length measurement system dominating the uncertainty. Pulse-to-pulse and day-to-day variations in the bunch length were found to be small compared to this [16].

\section{COLLIMATOR INSERTIONS}

The first set of collimator insertions were designed to maximize transverse geometric wakefields and to minimize resistive-wall wakes. All four insertions were fabricated from oxygen-free electronic grade copper, with a nominal conductivity of $5.85 \times 10^{7} \Omega^{-1} \mathrm{~m}^{-1}$ at $293.15 \mathrm{~K}$ [17]. The collimators are shown schematically in Fig. 3: three of the collimators are flat, while one of the collimators has a pyramidal taper to a square hole in order 


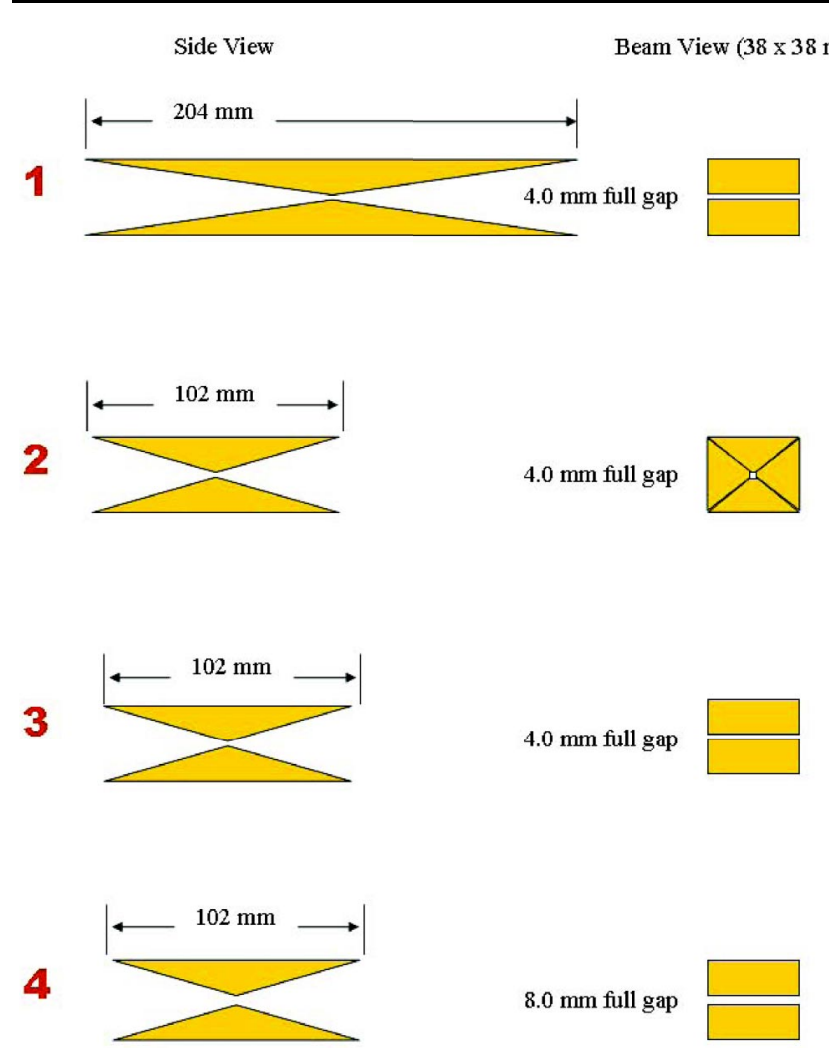

FIG. 3. (Color) Schematic side and end-on views of the test apertures used in collimator wakefield measurements.

to approximate a round collimator. None of the collimators contains an untapered section.

Collimator parameters are reviewed in Table I. It is clear from the analytic models that the transverse wakefields from the collimator insertions will be completely dominated by geometric wakefields. The taper regime of the collimators is rather ambiguous, as indicated by the 4th column of Table I. Since we expect that the diffractive wake kick expressions form an upper bound on the kick for a collimator with a given gap, we assume that the diffractive estimate will be the better one in any case where the inductive regime predicts a larger kick than the diffractive. In this experiment, this is always true except for estimates of the kicks from collimators 1 and 2 for long ( $1 \mathrm{~mm} \mathrm{rms})$ bunches; in these latter cases, the inductive kick is expected to be the better estimate. In Table I, the best estimate of the correct kick is in each case shown in bold. Note, however, that, because the collimator parameters turned out to be in the regime which is intermediate between the various asymptotic limits indicated in IB, we should not expect a good agreement between the analytical predictions and the experiment. For all diffractive wake kick estimates we use the $L_{F} \rightarrow 0$ limits from IB.

\section{WAKEFIELD MEASUREMENTS}

After positioning the desired test aperture in the beam path using the horizontal translation stage, the short range transverse wakefield is studied by moving the collimator test box vertically and measuring the angular deflection of the electron beam passing through the aperture as a function of the displacement between the beam axis and the collimator axis.

The deflection is measured by reconstructing the beam orbit based on the information from 5 BPMs located upstream from the test box and 32 BPMs downstream. At the beginning of each collimator measurement, a "reference orbit" is obtained by reading out the 37 BPMs for 100 pulses and averaging; during this time, the collimator is held stationary. Once a reference orbit is obtained, the collimator's vertical position is scanned: a typical scan involves moving the test aperture from approximately $1.4 \mathrm{~mm}$ below the nominal vertical position of the apparatus to $1.4 \mathrm{~mm}$ above the nominal position in steps of $0.2 \mathrm{~mm}$. At each collimator vertical position, the beam position reading on each BPM is recorded for 50 beam pulses; under normal operating conditions only a few seconds are required at each position setting of the apparatus. In total, a single measurement of the wakefield of a single collimator includes position measurements from 750 pulses obtained during the position scan as well as from 100 pulses during the stationary "reference orbit" data acquisition.

After the complete set of orbit data for one collimator is completed, the reference orbit is subtracted from the data taken during the vertical position scan, in order to suppress the DC offsets of the BPMs. The resulting differential BPM

TABLE I. Parameters of the first set of collimator test apertures. For all flat collimators, $h=$ $19 \mathrm{~mm}$. Expressions for diffractive and inductive kick factors $\kappa_{D}, \kappa_{I}$, can be found in Sec. IB [(Eqs. (3), (5), (8), and (9)]. rms bunch length is assumed to be $\sigma_{z}=0.5 \mathrm{~mm}$; results for $\sigma_{z}=$ $1 \mathrm{~mm}$ are also given, in parentheses. The expected best estimate of the wake kick is in all cases given in bold.

\begin{tabular}{lcccccc}
\hline \hline Slot & Type & $b_{1}, \mathrm{~mm}$ & $\theta_{T}, \mathrm{mrad}$ & $\alpha$ & $\kappa_{D}, \mathrm{~V} / \mathrm{pC} / \mathrm{mm}$ & $\kappa_{I}, \mathrm{~V} / \mathrm{pC} / \mathrm{mm}$ \\
\hline 1 & Flat & 2.0 & 165 & $0.66(0.33)$ & $\mathbf{2 . 2 5}(2.25)$ & $2.47(\mathbf{1 . 7 4})$ \\
2 & Square & 2.0 & 322 & $1.29(0.64)$ & $\mathbf{1 . 8 4}(1.84)$ & $2.93(\mathbf{1 . 4 6})$ \\
3 & Flat & 2.0 & 322 & $1.29(0.64)$ & $\mathbf{2 . 2 5}(\mathbf{2 . 2 5})$ & $3.45(2.44)$ \\
4 & Flat & 4.0 & 286 & $2.29(1.14)$ & $\mathbf{0 . 5 6}(\mathbf{0 . 5 6})$ & $1.15(0.81)$ \\
\hline \hline
\end{tabular}




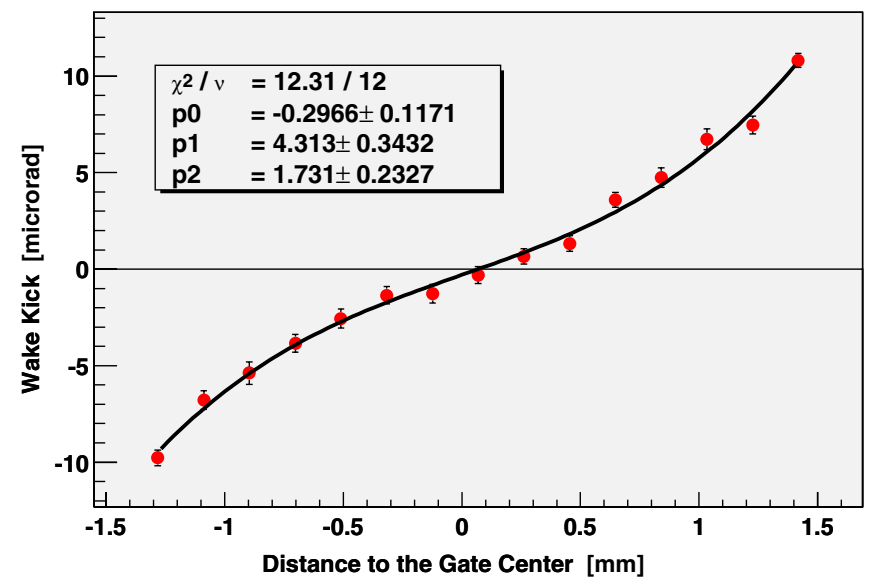

FIG. 4. (Color) Measured wakefield kicks for the slot 3 collimator as a function of collimator vertical position (red dots); 3 parameter fits (DC offset, linear, cubic) to the data (black line).

readings are then fitted to the beam transport model of the linac; the incoming beam position, incoming beam angle, and the collimator kick angle are the free parameters of the fit. The fit is performed independently on each pulse to minimize the impact of pulse-to-pulse jitter and to permit elimination of individual "noisy" data elements on each linac pulse. Selection criteria based on fit quality, BPM readings range, and beam current before and after the test box allow exclusion of poor quality bunches as well as those that follow erroneous trajectories. The accuracy of orbit reconstruction is verified by applying the same procedure to undisturbed bunches with similar incident beam parameters but no test aperture in the beam path. Figure 4 shows a typical example of a measurement. Statistical accuracy of approximately 0.5 microradian in the beam angular deflection measurement can be achieved using 50 pulses per collimator position.

The collimator wakefield is deduced by studying the correlation in the fitted wake kick with the position of the vertical translation stage. As shown in Fig. 4, the chosen range of vertical positions is such that the deflection of the beam enters the nonlinear regime of the collimator wakefield. For this reason, a three parameter cubic polynomial fit is used to extract the linear and cubic dependence on the position as well as the offset between the nominal vertical position of the apparatus and the position at which the beam is centered in the collimator; this offset is manifest as an offset in the symmetry point of the deflection curve. Several separate scans of the collimator position are performed and analyzed independently, and the results of the analyses are averaged to estimate the collimator kick factors.

For comparison with the theory and numerical simulations, wakefield strength is expressed in terms of a kick factor $\kappa$ for a Gaussian bunch:

$$
\kappa=\frac{E}{Q} \frac{y^{\prime}}{y_{0}}
$$

where $y^{\prime} / y_{0}$ is the measured linear component of the angular deflection dependence on the beam offset from the collimator axis for small offsets, $Q$ is the charge of the bunch, and $E$ is the beam energy. The bunch charge is monitored continuously by toroidal current monitors, and the bunch length is checked with a microwave bunch length monitor before each series of measurements.

An attempt was made to study the wakefield dependence on the bunch length by altering the bunch compressor settings. It has been found that changing the compression

TABLE II. Results of wakefield measurements and simulations for different bunch lengths. Values shown include the theoretical kick factor based on analytic expressions $\left(\kappa_{t}\right)$, measured kick factor $\left(\kappa_{m}\right)$, and kick factor from MAFIA or ECHO3D simulations $\left(\kappa_{\mathrm{MAFIA}}\right.$, $\left.\kappa_{\mathrm{ECHO}}\right) .(+)$ and $(-)$ indicate measurements with overcompressed and undercompressed beam, respectively. Errors shown for these measurements are based only on the reproducibility of measurements for each collimator at each compressor setting. Theoretical kick factors are based on the tentative selection of taper regime shown in Table I.

\begin{tabular}{cccccc}
\hline \hline Slot & Bunch length, $\mathrm{mm}$ & $\kappa_{t}, \mathrm{~V} / \mathrm{pC} / \mathrm{mm}$ & $\kappa_{m}, \mathrm{~V} / \mathrm{pC} / \mathrm{mm}$ & $\kappa_{\mathrm{MAFIA}}, \mathrm{V} / \mathrm{pC} / \mathrm{mm}$ & $\kappa_{\mathrm{ECHO}}, \mathrm{V} / \mathrm{pC} / \mathrm{mm}$ \\
\hline \multirow{2}{*}{1} & 0.5 & 2.3 & $1.3 \pm 0.1$ & 1.11 & 1.30 \\
& $1.0(+)$ & 1.7 & $0.8 \pm 0.1$ & 0.81 & 0.90 \\
2 & $1.0(-)$ & 1.7 & $0.8 \pm 0.1$ & 0.81 & 0.90 \\
\hline & 0.5 & 1.8 & $1.3 \pm 0.1$ & $\ldots$ & 1.57 \\
& $1.0(+)$ & 1.5 & $1.2 \pm 0.1$ & $\ldots$ & 1.31 \\
\hline & $1.0(-)$ & 1.5 & $1.3 \pm 0.1$ & $\cdots$ & 1.67 \\
& 0.5 & 2.3 & $1.3 \pm 0.1$ & 1.43 & 1.28 \\
& $1.0(+)$ & 2.3 & $1.1 \pm 0.1$ & 1.29 & 0.48 \\
4 & $1.0(-)$ & 2.3 & $1.0 \pm 0.1$ & 1.29 & 0.39 \\
& 0.5 & 0.56 & $0.54 \pm 0.05$ & 0.42 & 0.39 \\
\hline \hline
\end{tabular}


not only increased the bunch length but also degraded the beam quality significantly. The degraded beam quality was indicated by increased variability in the results from measurement to measurement, as well as an increase in the number of BPMs and pulses which were flagged as unacceptably noisy and removed from the main fit. In addition, the reproducibility of kick factor measurements made in a short time was found to be poor relative to measurements made at the minimum bunch length. Finally, it is suspected that the longitudinal distribution of the bunch is no longer Gaussian for under- and overcompressed bunches. Since the analytic expressions in Sec. IB assume a Gaussian longitudinal distribution, their validity for the under- and overcompressed bunches may be limited.

Table II compares the theoretical wakefield kick factors and those predicted by finite-element electromagnetic simulations with the measured kick factors at several bunch lengths. The results for both undercompressed and overcompressed beam with an estimated bunch length of approximately $1.0 \mathrm{~mm}$ (compared to $0.5 \mathrm{~mm}$ for nominal beam) are listed. Each measured wakefield kick factor is the average of several measurements, as described above, and the uncertainties are based on the reproducibility of the measurements and not on the estimated fit precisions from individual measurements. The theoretical estimates are all based on relations which are valid only for Gaussian longitudinal distributions, which implies that they may not be fully valid for the $1.0 \mathrm{~mm}$ bunch lengths due to possible changes in the bunch distribution for incomplete compression.

As a cross-check, a series of measurements was performed using the positron instead of the electron beam. While precise numerical comparison with the electron beam data was impossible due to lack of accurate bunch length and charge monitoring for the positron beam, the results showed good qualitative agreement.

\section{COMPARISON OF EXPERIMENT, THEORY, AND SIMULATION}

The measured kick factors for the collimators used in this experiment are compared to the theoretical expectations in Table II. For the flat collimators in slots 1, 3, and 4, there is qualitative similarity between the theoretical predictions and the measurements. Specifically, the theory predicts that the slot 1 collimator will be the most sensitive to bunch length; that the wakefield kicks from the slot 1 and the slot 3 collimators will be comparable; and that the wake kick of the slot 4 collimator will be the one in closest agreement with the diffractive estimates, since it is furthest into the diffractive regime. All of these predictions are supported by the measurements. The most obvious discrepancy between theory and measurement is that the predicted wake kicks for the collimators in slots 1 and 3 are almost a factor of 2 larger than what was measured. This is not surprising, given that the applicability criterion for use of the diffractive regime wake kick relations is $\alpha \gg 1$, whereas the actual value of $\alpha$ for these measurements is between 0.64 and 1.29. Interestingly, in the case of $1 \mathrm{~mm}$ rms bunch length and the slot 1 collimator, the applicability criteria for Eq. (5) appears to be reasonably well met, yet the ratio between the measured and predicted wake kicks in this case is within errors of the ratio for the $0.5 \mathrm{~mm}$ bunch, for which the prediction was made using Eq. (8). The agreement of measurements and Eq. (8) is much better for slot 4 even at $1 \mathrm{~mm} \mathrm{rms}$ bunch length $(\alpha=1.14)$ than it is for slot 3 at $0.5 \mathrm{~mm}$ rms bunch length $(\alpha=1.29)$. This suggests that the applicability criteria may be more complex than our current understanding would indicate.

In the case of the square collimator in slot 2, the quantitative agreement between theory and measurement is better than in the case of the comparable rectangular collimator in slot 3. The theoretical expectation of weak but measurable dependence on bunch length is not observed in the data.

\section{A. Numerical simulations of collimator wakefields}

In addition to the theoretical predictions of the kick factors, numerical estimates of the kick factors were obtained from the simulation programs MAFIA [18] and ECHO3D [19], and these estimates are also included in Table II.

The MAFIA calculations of the wakefields were performed in 3D. Making use of symmetry, one-quarter of each structure was simulated. For each calculation, two runs were done using different boundary conditions at the symmetry plane that separates the top and bottom parts of the collimator. The results were subtracted between the two runs to give the total transverse wakefield, including dipole, quadrupole, and higher order contributions. The expected accuracy of the MAFIA calculations is $5 \%$.

The ECHO3D calculations were performed in 3D. Calculations for complete collimator structures were performed, as well as calculations which used one-quarter of each structure and made use of the collimator symmetries. In all cases, dipole and quadrupole wakefield contributions were included. The expected accuracy of the ECHO3D calculations is $1 \%$.

In the case of the slot 1 collimator, the agreement between ECHO3D and the measurements is within experimental error for all bunch lengths. In all other cases, the simulations produce estimates for the wakefield kicks which are approximately correct, with agreement at the level of $20 \%-30 \%$ in the worst cases, but there is not a clear and consistent pattern of predictions from the simulations falling within the uncertainties of the measurements other than the slot 1 collimator and ECHO3D.

\section{CONCLUSIONS}

The transverse wakefields from collimators present a potentially serious challenge to linear colliders, which require an extraordinary beam quality in order to deliver 
luminosity. In order to improve understanding of this phenomenon, we have constructed a beam test apparatus for directly measuring the wakefields of collimators, and used the apparatus to measure the wakefield deflections from 4 sets of longitudinally tapered collimators acting on the beam in the SLAC linac.

In the case of the flat collimators, qualitative agreement between theory and measurement was observed, but in many cases there was a quantitative discrepancy as large as a factor of 2 . The quantitative agreement between theory and measurement was somewhat better for the square collimator, but there was a qualitative prediction of weak but measurable dependence on bunch length which was not observed in the measurements.

For all 4 collimators, numerical electromagnetic simulations were able to predict the measured wakefield kick with typical agreement at the level of $20 \%$. In a few cases the measurements and the simulations disagreed at the level of $30 \%$, while in some cases the agreement was quite good. The simulations also reproduced the observed weak dependence on bunch length.

The process of reconciling theory, simulation, and experiment in the realm of collimator wakefields is still in its infancy. In the future, we plan to perform additional experiments which will probe the parameter space of geometric wakefields more thoroughly. In addition, experiments which study the resistive-wall and/or surface-roughness contributions to collimator wakefields are planned.

\section{ACKNOWLEDGMENTS}

The collimator wakefield apparatus was designed by B. Brugnoletti, and constructed and installed by expert teams in the SLAC Technical Division. D. Burke of SLAC provided invaluable guidance and advice on both experimental and technical issues. T. Anderson of SLAC assisted with the production of graphics for some of the figures. As with all accelerator experiments at SLAC, the support of the Accelerator Department in integrating the apparatus and the experiments into the existing infrastructure and schedule of the laboratory were essential. The work described in this paper was performed with support from the United States Department of Energy, Contracts No. DE-AC02-76SF00515 and DE-AC03-76SF00515.

[1] P. Tenenbaum, E. Doyle, J. Frisch, R. Helm, S. Hertzbach, L. Keller, T. Raubenheimer, and M. Woodley, SLAC, Tech. Rep. SLAC-TN-03-069, 2001.

[2] C. Adolphsen et al., Zeroth-Order Design Report for the Next Linear Collider (Stanford University, Stanford, 1996), Chap. 9, pp. 555-642.

[3] K. Bane, C. Adolphsen, F. Decker, P. Emma, P. Krejcik, and F. Zimmermann, in Proceedings of the 16th Particle Accelerator Conference (1995).

[4] F. Zimmermann, K. Bane, and C. Ng, in Proceedings of the 5th European Particle Accelerator Conference (1996).

[5] F. Zimmermann, T. Usher, P. Tenenbaum, P. Raimondi, P. Emma, K. Bane, and F. Decker, SLAC, Tech. Rep. SLAC-CN-415, 1997.

[6] K. Yokoya, CERN, Tech. Rep. CERN SL/90-88 (AP), 1990.

[7] G. Stupakov, Part. Accel. 56, 83 (1996).

[8] G. Stupakov, SLAC, Tech. Rep. SLAC-PUB-7167, 1996.

[9] G. Stupakov, in Proceedings of the Particle Accelerator Conference (2001).

[10] S. Krinsky, Phys. Rev. ST Accel. Beams 8, 124403 (2005).

[11] E. Gianfelice and L. Palumbo, IEEE Trans. Nucl. Sci. 37, 1084 (1990).

[12] I. Zagorodnov and K. Bane, in Proceedings of the European Particle Accelerator Conference (2006).

[13] K. Bane, G. Stupakov, and I. Zagorodnov (unpublished).

[14] G. Bowden, P. Holik, S. Wagner, G. Heimlinger, and R. Settles, Nucl. Instrum. Methods Phys. Res., Sect. A 368, 579 (1996).

[15] R. Akre, L. Bentson, P. Emma, and P. Krejcik, in Proceedings of the Eighth European Particle Accelerator Conference (2002).

[16] H. Schlarb (private communication).

[17] CRC Handbook of Chemistry and Physics, edited by R. Weast (CRC Press, Boca Raton, FL, 1984), p. E-78, 65th ed.

[18] R. Klatt et al., in Proceedings of the 1986 International Linac Conference (1986), p. 276.

[19] I. Zagorodnov and T. Weiland, Phys. Rev. ST Accel. Beams 8, 042001 (2005). 